polymer substrates. The type of conductive filler considerably determines the characteristics of nucleation, growth and the electroforming of the product microstructures.

The development of an ideal 2-component combination, also besides the commercially available polymers, is necessary. Here, also the use of polymer material, which is intrinsically conductive because of the chemical polymer structure, will promise interesting results.

A further important part of investigations will be the production of metallic mold inserts for the injection molding of templates of the important hole type and the following electroforming and replication.

Electrochemical investigations like voltamograms $i=f(E)$ and transients $i=f(t)$ on the properties of conductive polymers will exhibit detailed qualitative and quantitative information about the processes at the interface polymer-electrolyte.

Received: November 10, 2003 Final version: March 04, 2004

[1] V. Piotter, N. Holstein, L. Merz, R. Ruprecht, J. Hausselt, Proc. of $3^{\text {rd }}$ Euspen International Conference Eindhoven 2002, 337-340.

[2] G. Baumeister, N. Holstein, V. Piotter, R. Ruprecht, G. Schanz, M. Guttmann, A. Häfele, FZK Nachrichten 2002, 34/2-3, 198-210.

[3] W. Michaeli, A. Rogalla: FEM Feinwerktechnik 1996, 104/ 9, 641-643.

[4] M. Guttmann, E. Walch, G. Schanz, J. Konys, Galvanotechnik 2000, 54/11, 16-28, \& 2001, 55/1, 34-36.

[5] K. Leyendecker, W. Bacher, K. Bade, W. Stark, FZKA Bericht 5594, 1995.

[6] V. Piotter, R. Ruprecht, J. Schrök, J. H. Haußelt, Jahrbuch Oberflächentechnik 1996.

[7] M. Harmening, W. Ehrfeld, KfK-Bericht 4711, 1990.

[8] H. Vollmer, W. Ehrfeld, P. Hagmann, KfK-Bericht 4267, 1987.

[9] P. Hagmann, E. Becker, A. Maner, W. Ehrfeld, D. Müchmeyer, Patent EP 0331208, Kernforschungszentrum Karlsruhe, 1987.

[10] V. Piotter, N. Holstein, E. Oskotski, R. Ruprecht, Abschlussbericht zum DFG-Vorhaben PI 363/2-1 und PI 363/2-2, DFG-SPP 1012: "Mikromechanische Produktionstechnik", Juli 2003.

[11] V. Piotter, N. Holstein, E. Oskotski, G. Schanz, R. Ruprecht, J. Hausselt, Proc. of $4^{\text {th }}$ Euspen International Conference Aachen 2003, 367-370.

[12] N. Holstein, V. Piotter, G. Schanz, R. Ruprecht, Proc. MICRO System Technolgies 2003, 273-280.

\section{Comparison of Single Crystal Simple Shear Deformation Experiments with Crystal Plasticity Finite Element Simulations**}

\author{
By Franz Roters, Yanwen Wang, Jui-Chao Kuo, \\ and Dierk Raabe*
}

This article presents an investigation on the evolution of the crystallographic texture and of the equivalent strain distribution during simple shear deformation of a pure Al single crystal. Previous studies in which we presented detailed comparisons between crystal plasticity finite element simulations and corresponding experiments under plane strain load revealed in part a disturbing influence of friction on the results. ${ }^{[1-6]}$ This effect impedes the validation of advanced crystal plasticity finite element methods.

Friction between the tool and the specimen introduces two main problems into such tests rendering validation efforts a difficult task: First, it introduces additional shear stress components of unknown magnitude. Second, its magnitude changes during loading in an unknown fashion as deformation proceeds. Hence, a more quantitative approach lies in using simple shear deformation experiments since they do not involve friction.

The structure of this article is as follows: First, we provide a concise review of earlier work on simple shear experiments. Second, we describe the experimental procedure. Third, we give a brief introduction to the model approach and explain how we determined the parameters of the underlying constitutive model. Fourth, we compare the experimental observations with the model predictions in terms of the texture evolution and the strain distribution.

[*] Dr. F. Roters, Dr. J.-C. Kuo, Prof. D. Raabe Max-Planck-Institut für Eisenforschung Max-Planck-Str. 1, 40237 Düsseldorf, Germany E-mail: raabe@mpie.de

Dr. Y. Wang

Department of Mechanics

University of Missouri-Rolla, USA

[**] We are grateful to M. Winning and G. Gottstein at the Institut für Metallkunde und Metallphysik, RWTH Aachen, Germany, for providing the Al single crystals. Funding by the Deutsche Forschungsgemeinschaft DFG within the Schwerpunktprogramm 1138 (Modellierung von Größeneinflüssen bei Fertigungsprozessen) is gratefully acknowledged. 
Related studies on simple shear plasticity tests: Shear deformation experiments have been widely discussed in the crystal plasticity literature since they offer a loading mode without friction. ${ }^{[7-9]}$ For instance, Rauch ${ }^{[7]}$ discussed the simple shear test with respect to the analysis of in-plane plastic anisotropy parameters. Horstemeyer et al. ${ }^{[8,9]}$ used three modeling frameworks (molecular dynamics, crystal plasticity FEM, internal state variable formulation in conjunction with FEM) for predicting simple shear deformation of Nickel. They found ${ }^{[9]}$ that the aspect ratio of the specimen (length-to-height) has a strong influence on the stress distribution inside the specimen during shear deformation. Extreme cases occur for samples which have a very large aspect ratio (true simple shear) as opposed to those with a small one (bending). An aspect ratio of one leads to a multiaxial response. Tugcu et al. ${ }^{[10]}$ used constrained simple shear tests to investigate the predictive capabilities of 3D anisotropic yield criteria. Lopes et al. ${ }^{[11]}$ used simple shear experiments for investigating the strain hardening anisotropy of aluminium and compared it with that during uniaxial tension. Following the results discussed in these articles we use an aspect ratio of $\approx 1.4$ for the shape of the single crystal sample before loading.

Experimental: A single crystal $(99.999 \% \mathrm{Al})$ with a starting orientation of $\varphi_{1}=3.4^{\circ}, \phi=37.6^{\circ}, \varphi_{2}=36.5^{\circ}$ (Bunge Euler angles, the reference system is given by the longitudinal and transverse directions, respectively) was produced using a modified Bridgman technique. The simple shear experiment was conducted at a constant shear rate of $10^{-6} \mathrm{~s}^{-1}$ using the setup shown in Figure 1. The in-plane distribution of the accumulated von Mises equivalent plastic strain was determined on the sample normal surface using photogrammetry ${ }^{[2,12]}$. The strain was measured in a series of consecutive displacement steps of $0.1 \mathrm{~mm}$ each, up to a total shear displacement of $1.3 \mathrm{~mm}$. Each measurement covered an area of $3.1 \times 2.2 \mathrm{~mm}^{2}$. Crystal orientation maps were taken at the sample normal surface before and after shear deformation using EBSD.

Simulation procedure: The constitutive model used for the crystal plasticity finite element simulations is formulated in accord with the work of Kalidindi and Anand. ${ }^{[13-15]}$ The con-



Fig. 2. The experimental shear stress versus shear strain data and its simulated counterpart.

stitutive equations were implemented into the commercial finite element code MSC.Marc200x by means of the user defined material subroutine HYPELA2. ${ }^{[16]}$ We use a 3D finite element mesh with 1680 eight-node brick elements for the discretization of the sample. As aluminium is a fcc material we use the twelve $\{111\}<110>$ slip systems for the simulation. $\overline{\gamma_{0}}=0.001 \mathrm{~s}^{-1}$ was used as reference value for the slip rate and the strain rate sensitivity parameter $m$ is taken as 0.02 . The hardening matrix parameters are $q^{\alpha \beta}=1.0$ for coplanar slip systems and $q^{\alpha \beta}=1.4$ for non-coplanar systems. The components of the elasticity tensor (pure single crystalline $\mathrm{Al}$ ) are taken as $C_{11}=106.75 \mathrm{GPa}, C_{12}=60.41 \mathrm{GPa}$, and $C_{44}=$ 28.34 GPa. The rest of the constitutive parameters was identified by gradually approximating them in the finite element simulation until an optimal match was obtained between the experimental and the simulated stress-strain curves (Fig. 2). By using this method we obtained as constitutive values $s_{0}=$ 12.5 MPa for the initial slip resistance, $s_{s}=75 \mathrm{MPa}$ for the saturation slip resistance, and $h_{0}=60 \mathrm{MPa}$ and $\mathrm{a}=2.25$ for the hardening parameters (see refs. $1-6$ for constitutive details).

Results and discussion: Figure 3 shows the evolution of the crystallographic texture during shear in terms of $\{111\}$ pole

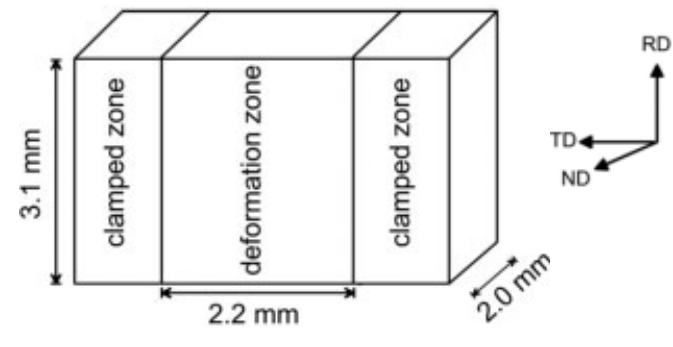

Fig. 1. Schematic drawing of the experimental set-up.

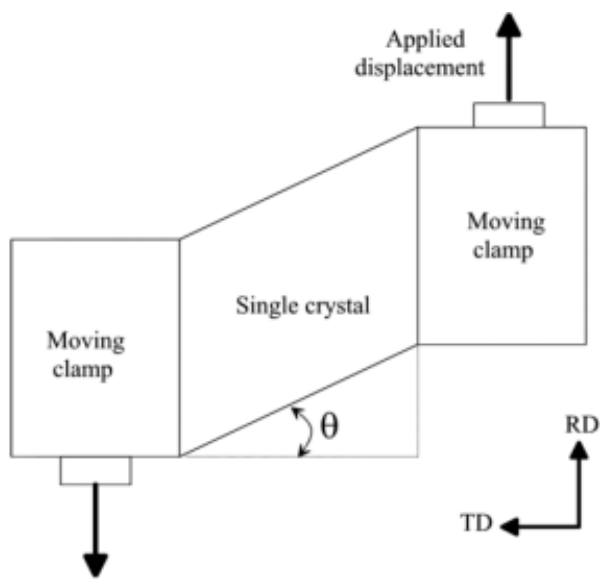



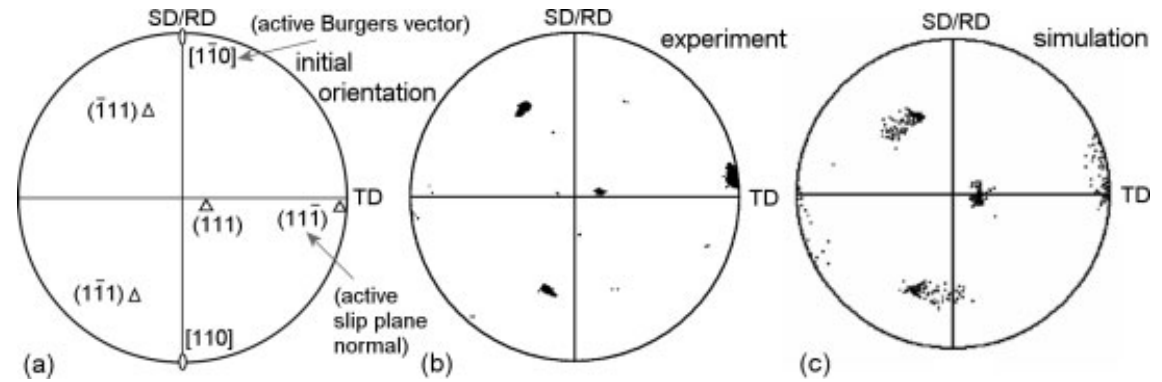

(c)

Fig. 3. \{111\} pole figures, SD/RD: shear / longitudinal direction; TD: transverse direction. (a) starting orientation of the single crystal before deformation, active slip system; (b) after shearing, experiment; (c) after shearing, simulation.

figures. Both, experiment and simulation reveal lattice rotations essentially (but not exactly) about the normal direction during deformation. Schmid analysis shows that the (111) [11̄0] slip system experiences the maximum resolved shear stress and is, hence, the predominant slip system. This is also correctly predicted by the finite element simulation.

Figure 4 shows the simulated accumulated plastic shear on all slip systems. The data show nearly single-slip behaviour with a strong dominance of glide on (111) [11̄0]

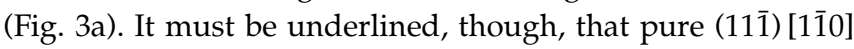
slip in an ideally oriented crystal (meaning that the (111) slip plane would be exactly parallel to the shear direction) would not entail any change in the lattice orientation but only a pure shape change. This means that only the fact that the starting crystal had a small deviation from this exact position entails a lattice reorientation (Fig. 3).

The fact that the simulation shows a somewhat larger scatter of the orientations than the experiment is most likely due to the ideal boundary conditions prescribed in the simulation. Such a strict boundary conditions does not allow any deviation from ideal simple shear on the contact areas between the specimen and the tool. In the experiments, although clamped tightly as indicated in the drawing shown in Figure 1, small deviations are always possible between the tool and the sample.

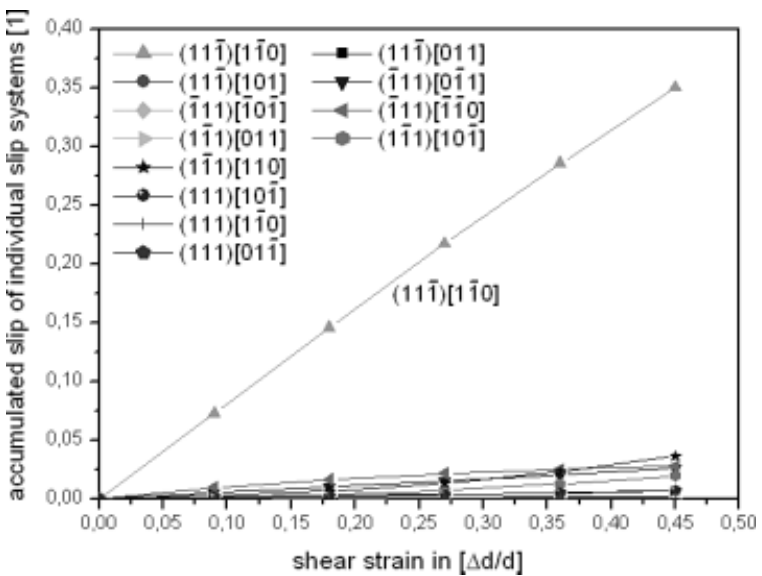

Fig. 4. The accumulated slip of the individual slip systems during simple shear deformation, simulation result.
Figure 5 shows the evolution of the in-plane lateral arrangement and magnitude of the von Mises equivalent strain (engineering notation) during shearing as observed in experiment and as predicted by simulation. The predicted data reveal excellent quantitative agreement with the measurements.

An important result of the strain measurement is that the simple shear test is indeed much more homogeneous than the lubricated plane strain tests, which are commonly used for crystal plasticity experiments (see for comparison data in Raabe et al. ${ }^{[1]}$ Sachtleber et al., ${ }^{[2]}$ and Raabe et al. ${ }^{[5]}$ ). Concerning the macroscopic boundary conditions we attribute this result to the absence of friction. Concerning the microscopic boundary conditions we suppose that the observed strain homogeneity is in part also due to the fact that the crystal is deformed basically by single slip. The small normal rotation which takes place during shearing does not lead to substantial heterogeneity. These points underline that mechanical shear tests offer an excellent approach for conducting crystal plasticity experiments under well defined conditions and without friction qualifying them
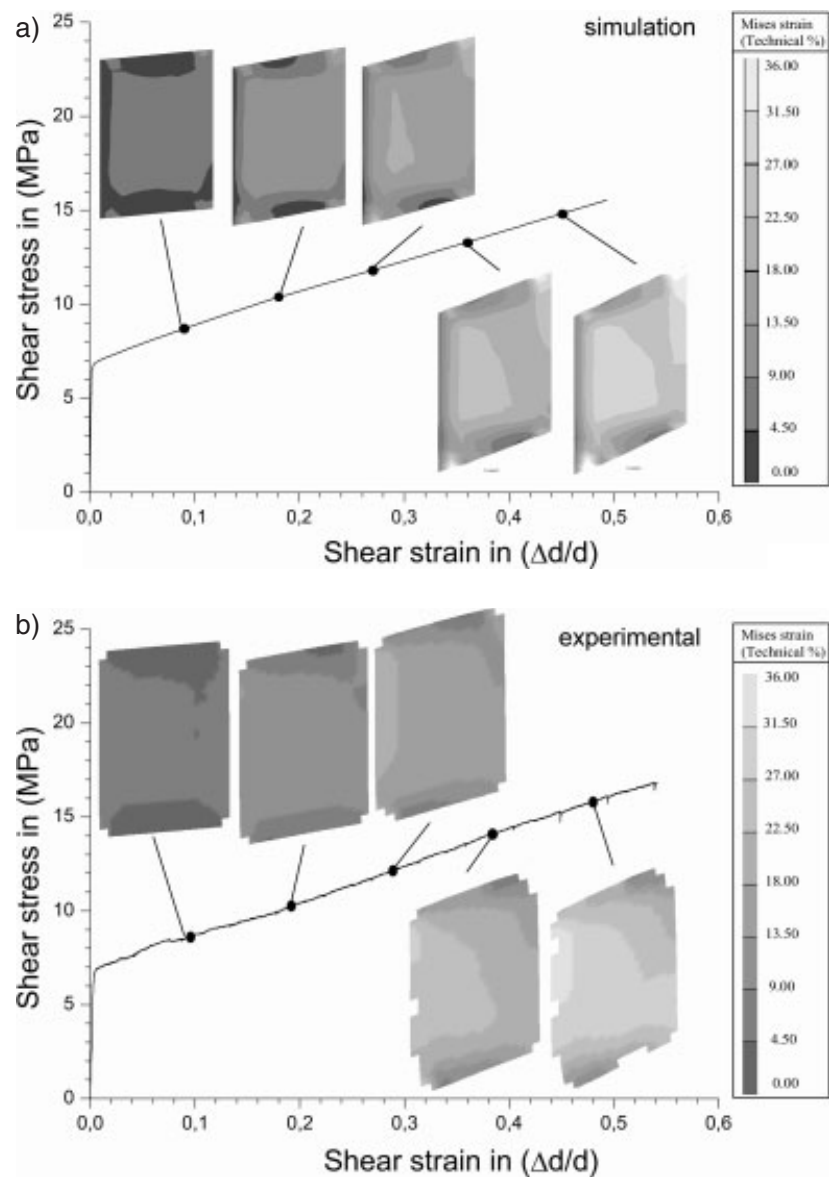

Fig. 5. Von Mises strain distribution during single crystal simple shear, (a) experimental (top); (b) simulation (bottom). 
as a good choice for the experimental validation of crystal plasticity finite element simulations.

Conclusions: We presented a study about the evolution of texture and accumulated plastic equivalent strain in an $\mathrm{Al}$ single crystal during simple shear deformation using experiment and crystal plasticity finite element simulations. The main findings are:

- The crystal deforms nearly by single slip. Other systems have a very small contribution to the overall slip rate.

- The crystal undergoes a small lattice rotation about the normal direction during shearing. This rotation is due to the small deviation of the initial orientation from the ideal single slip orientation.

- The observed surface strain patterns show that the shear test was very homogeneous.

- Both, the simulated textures and von Mises strain patterns are in good agreement with those observed in experiment.

We suggest that (simple) shear tests are more suited for the validation of crystal plasticity finite element (or other similar) models than channel die tests (suffer from friction) or tension tests (suffer from necking).

Received: April 24, 2004

Final version: May 12, 2004

[1] D. Raabe, M. Sachtleber, Z. Zhao, F. Roters, S. Zaefferer, Acta Mater. 2001, 49, 3433.

[2] M. Sachtleber, Z. Zhao, D. Raabe, Mater. Sci. Eng. 2002, A336, 81.

[3] D. Raabe, F. Roters, Intern. J. Plast. 2004, 20, 339; D. Raabe, M. Sachtleber, H. Weiland, G. Scheele, Z. Zhao, Acta Mater. 2003, 51, 1539.

[4] S. Zaefferer, J.-C. Kuo, Z. Zhao, M. Winning, D. Raabe, Acta Mater. 2003, 51, 4719.

[5] D. Raabe, Z. Zhao, F. Roters, S. Zaefferer, Scripta Mater. 2004, 50, 1085.

[6] E F. Rauch, Mater. Sci. Eng. 1998, A241, 179.

[7] M. F. Horstemeyer, M. I. Baskes, S. J. Plimpton, Acta Mater. 2001, 49, 4363.

[8] M. F. Horstemeyer, M. I. Baskes, V. C. Prantil, J. Philliber, S. Vonderheide, Model. Sim. Mater. Sci. Eng. 2003, 11, 265.

[9] P. Tugcu, P. D. Wu, K. W. Neale, Intern J. Plast. 2002, $18,1219$.

[10] A. B. Lopes, F. Farlat, J. J. Gracio, J. F. F. Duarte, E. F. Rauch, Intern. J. Plast. 2003, 19, 1.

[11] Handbook for the Aramis system by GOM mbh, Gesellschaft für Optische Meßtechnik, Version September 2000, Braunschweig, Germany.

[12] S. R. Kalidindi, C. A. Bronkhorst, L. J. Anand, Mech. Phys. Sol. 1992, 40, 537.

[13] S. R. Kalidindi, L. J. Anand, Metall. Transact. 1993, A24, 989.
[14] S. R. Kalidindi, S. E. Schoenfeld, Mater. Sci. Eng. 2000, A293, 120.

[15] MSC. Marc User's Manual 2001, Vol. D, MSC Software Corporation.

\section{Technical Cost Modeling for the Mechanical Milling at Cryogenic Temperature (Cryomilling)}

\author{
By Jichun Ye and Julie M. Schoenung
}

Our world is a world of materials, and materials scientists and engineers never stop pursuing novel materials with greatly improved properties and performance to meet the ever-increasing demands in a broad range of fields. Since the pioneering work of Gleiter and coworkers in the 1980s, ${ }^{[1]}$ nanophase materials and nanocomposites, characterized by the microstructural dimensions less than $100 \mathrm{~nm}$, have received continuously increasing attention due to their superior physical and mechanical properties. Although nanostructured materials have demonstrated the potential to be widely used as functional as well as structural materials, it is an everlasting problem to fabricate bulk nanostructured materials on the order of at least a cubic centimeter in size, as would be necessary for many structural applications. To date, there are few fabrication processes that can be used to synthesize bulk materials in these dimensions. ${ }^{[2]}$ Cryomilling, mechanical milling at cryogenic temperature, is one of the few processing approaches available for the fabrication of large quantities of nanostructured materials, thereby making it possible for nanostructured materials to be commercialized. ${ }^{[3]}$ Several researchers have found that the bulk nanostructured metals, ${ }^{[3]}$ metal matrix nanocomposites, ${ }^{[4]}$ and intermetallic compounds ${ }^{[5]}$ prepared by cryomilling and subsequent consolidation steps have shown greatly improved mechanical properties compared to the conventional counterparts. No matter how exciting and promising a technology is, its ultimate realization is invariably dependent on economic success. As a new and promising technique, the cost effectiveness of cryomilling and subsequent consolidation steps will determine the level of investment in this new industry. The revenues and profits should be foreseeable before manufacturers re-

[*] J. Ye, Prof. J. M. Schoenung

Department of Chemical Engineering and Materials Science, University of California, Davis, CA 95616-5294, USA E-mail: jmschoenung@ucdavis.edu

[**] Financial support was provided by the Office of Naval Research under contract N00014-03-C-0164. 